\title{
Altered vagal and intestinal mechanosensory function in chronic unexplained dyspepsia
}

\author{
G Holtmann, H Goebell, F Jockenhoevel, N J Talley
}

\begin{abstract}
Background-Abnormal visceral mechanosensory and vagal function may play a role in the development of functional gastrointestinal disorders.

Aims-To assess whether vagal efferent and afferent function is linked with small intestinal mechanosensory function.

Methods-In seven patients with functional dyspepsia, six patients with a history of Billroth I gastrectomy and/or vagotomy, and seven healthy controls, intestinal perception thresholds were tested by a randomised ramp distension procedure performed with a barostat device. On a separate day, an insulin hypoglycaemia test was performed to assess the plasma levels of pancreatic polypeptide (PP) in response to hypoglycaemia, as a test of efferent vagal function. Results-First perception of intestinal balloon distension occurred at significantly lower pressures in patients with functional dyspepsia (median 19.3, range 14.7-25.3 mm Hg) compared with healthy controls (median 26.0, range 21.7-43.7 mm Hg, p<0.01). Sensory thresholds were significantly lower in patients after gastrectomy (median 12.2 , range $8.0-14.7 \mathrm{~mm}$ $\mathrm{Hg}, \mathrm{p}<0.05$ versus all others). In healthy controls and patients with functional dyspepsia, insulin hypoglycaemia significantly $(p<0.001)$ increased plasma PP levels. However, only two out of seven patients with functional dyspepsia had a more than twofold increase in PP values whereas all healthy controls had a more than twofold increase in PP levels after insulin hypoglycaemia $(p<0.05)$. In contrast, there was no significant PP response in the gastrectomised patients (median $2 \%$, range -10 to $+23 \%$ ). PP responses and visceral sensory thresholds were significantly correlated $(r=0.65, p<0.002)$.
\end{abstract}

Conclusions-The diminished PP response after insulin hypoglycaemia indicates disturbed efferent vagal function in a subgroup of patients with functional dyspepsia. The data also suggest that the intact vagal nerve may exert an antinociceptive visceral effect.

(Gut 1998;42:501-506)

Keywords: functional dyspepsia; intestino-intestinal reflexes; gastrointestinal motility; pancreatic polypeptide

Altered visceral mechanosensory function (hyperalgesia) is believed to play a role in the development of symptoms in at least a subgroup of patients with otherwise unexplained (functional) abdominal complaints. ${ }^{1-5}$ The mechanisms involved in the development of visceral hypersensitivity are incompletely determined, but impaired central down regulation of visceral afferents or sensitisation by mucosal inflammation may be important. ${ }^{67}$

In healthy subjects, balloon distension in the duodenum results in gastric relaxation. ${ }^{8}$ This motor response to distension is impaired in patients with functional dyspepsia. ${ }^{9}$ Recently we have shown impaired small intestinal motor responses to duodenal distension in patients with functional dyspepsia. ${ }^{10}{ }^{11} \mathrm{~A}$ reduced release of pancreatic polypeptide (PP) after sham feeding has been observed in some patients with functional dyspepsia, implicating impaired efferent cholinergic vagal function. ${ }^{12}$ In addition, there is evidence that vagal function may be impaired in patients with irritable bowel syndrome (IBS), ${ }^{13}$ a condition that frequently overlaps with functional dyspepsia. ${ }^{14}$ The relation between altered mechanosensory function or alterations of motor abnormalities and efferent vagal function in humans has not been studied.

We wished to evaluate the potential role of the vagus nerve in the development of visceral hyperalgesia in patients with functional dyspepsia. Therefore, we aimed to: (1) determine as a test for vagal efferent function the PP response to insulin hypoglycaemia in patients with functional dyspepsia, patients with a Billroth I gastrectomy and vagal nerve transection, and healthy asymptomatic controls; (2) assess small intestinal mechanosensory thresholds in these groups; and (3) evaluate the association between the PP response following insulin induced hypoglycaemia and visceral sensory thresholds.

Material and methods

PATIENTS AND CONTROLS

Seven patients with functional dyspepsia were recruited for participation in this study (three females, four males, median age 36.5, range 19-53 years). In all patients, symptoms (postprandial fullness, early satiety, abdominal pain) occurred more than three times a week, and all patients experienced symptoms after a meal. Abdominal symptoms had been present for more than three years and treatment had yielded only temporary relief of symptoms. All patients had a normal physical examination and no alarm symptoms (for example, no weight loss). Laboratory testing (white and red blood count, sedimentation rate, fasting blood glucose level, liver function tests), abdominal

Medicine, Division

Essen, Germany. 
sonography, and upper gastrointestinal endoscopy were normal or negative. All patients over 45 years also had a colonoscopy performed. In addition, six patients with a history of Billroth I gastrectomy for duodenal ulcer disease were enrolled (two females, four males, median age 46.5 , range $41-52$ years). These patients were referred for upper endoscopy for various reasons (follow up surveillance more than 10 years after partial gastrectomy $(n=3)$, routine upper endoscopy prior to initiation of anticoagulation therapy $(n=2)$, and polypectomy for colonic polyps $(n=1))$. On specific questioning, all Billroth I patients reported meal related symptoms, but body weight was within the normal range. Charts and surgical reports of these patients were checked to ensure that they had undergone Billroth I gastrectomy with transection of vagal branches innervating the duodenum and the pancreas $(n=3)$ or Billroth I gastrectomy plus truncal vagotomy $(n=3)$. Surgical procedures were done at least three years prior to enrolment (range 3 to 15 years).

Furthermore, seven healthy subjects (four females, three males, mean age 34.6, range 22-56 years) without abdominal symptoms and without a history of previous gastrointestinal disorders were enrolled. These subjects were recruited by advertisement. Prior to enrolment they were screened in a standardised manner by means of the BDQ (Bowel Disease Questionnaire ${ }^{15}{ }^{16}$ ) for abdominal symptoms and had a complete physical examination. Only subjects without abdominal symptoms or evidence of organic disease were enrolled.

TUBE, BAROSTAT, AND DISTENSION PROCEDURE Duodenal motility was recorded by standard pneumohydraulic perfusion manometry. ${ }^{17}$ Pressure recording ports were located 5 and 10 $\mathrm{cm}$ distal to the bag as well as 14,16 , and $18 \mathrm{~cm}$ proximal to the barostat bag (Hefty Baggies, Mobil Chemical, Pittsford, New York). This infinitely compliant bag was firmly tied with silk over two steel rings that were glued $8 \mathrm{~cm}$ apart to the tube. These steel rings ensured that the lumens of the tubes were not occluded by the silk ligature. The attached bag had a maximal capacity of $200 \mathrm{ml}$. In previous studies ${ }^{10} 18$ we have shown that this size of bag is most appropriate for small intestinal distension experiments (hence the maximal capacity is clearly higher than the maximal tolerated volume). One additional line was attached to the tube for the intraduodenal nutrient infusion. The infusion port was located $8 \mathrm{~cm}$ above the barostat bag.

The distensions were performed with the ISOBAR 3 device ( $\mathrm{G} \& \mathrm{~J}$ Electronics, Ontario, Canada). The barostat was connected to the bag via two separate lines. One tube (inner diameter $1.6 \mathrm{~mm}$ ) was used to monitor the pressure inside the bag while the other (diameter $2.8 \mathrm{~mm}$ ) was used to inflate or deflate the bag. The maximum speed of the air pump was set to $25 \mathrm{ml} / \mathrm{s}$. Each distension procedure consisted of up to 25 steps (including the interposed pressure steps with lower pressures) and each pressure step lasted 15 seconds. The pressure level began at $5 \mathrm{~mm} \mathrm{Hg}$ and increased in increments of $2 \mathrm{~mm} \mathrm{Hg}$ up to a maximum pressure of $45 \mathrm{~mm} \mathrm{Hg}$ (at this pressure a safety loop was activated that prevented higher pressures for safety reasons). In random order, up to five steps with pressures $50 \%$ below the preceding step were interposed into the distension procedure. Thus for the volunteers it was unpredictable when a given pressure was reached. Immediately before starting the distensions, the volunteers and patients were informed that a distension procedure was to be performed and instructed to indicate the perceived intensity of the distension after a beep on a keypad. The buttons on the keypad were labelled 0 (no sensation), 1 (perception of the distension), and 2 (pain: terminate the distension). The subjects were instructed to press the appropriate button immediately after the beep that occurred when a given pressure level was reached; subjects were kept unaware of the pressure levels.

PROTOCOL

Following approval of the research protocol by the local ethics committee and informed consent, the subjects swallowed a six lumen orojejunal tube after an overnight fast. Under fluoroscopic guidance, the tube with the attached barostat was placed in the small intestine $30 \mathrm{~cm}$ distal to the pylorus or-in patients with gastrectomy $-30 \mathrm{~cm}$ distal to the anastomosis.

Thereafter, intestinal motility was recorded until a propagating phase III complex was observed or a maximum of 150 minutes of recording were completed. Thirty and $60 \mathrm{~min}$ utes after the duodenal phase III, the distension was performed with $2 \mathrm{~mm} \mathrm{Hg}$ pressure increments. In subjects without a phase III within 120 minutes after the start of motility recording, the intestinal distension procedure was performed and repeated after another 30 minutes. Thereafter, a liquid enteral feeding solution (Fresubin, Fresenius AG, Bad Homburg, Germany) with $55 \%$ of energy as carbohydrates, $30 \%$ as fat, and $15 \%$ as protein (osmolarity $300 \mathrm{mosmol} / \mathrm{l}$, energy density $0.006 \mathrm{MJ} / \mathrm{ml}$ ) was infused into the small intestine $10 \mathrm{~cm}$ orad to the distending bag at a rate of $2.5 \mathrm{ml} / \mathrm{min}$. Distensions were not performed after the start of the nutrient infusion.

INSULIN HYPOGLYCAEMIA

On a separate day, all subjects underwent a standardised insulin hypoglycaemia test. Recombinant human insulin was given intravenously at a dose of 0.2 IU per kg body weight and blood glucose measured at five minute intervals. Blood samples were taken before administration of insulin and at 15 minute intervals for up to 60 minutes. When blood glucose dropped below $1.93 \mathrm{~mol} / 1$ (35 mg/dl), glucose $(10 \% \mathrm{w} / \mathrm{w})$ was given intravenously to maintain blood glucose levels between 80 and $120 \mathrm{mg} / \mathrm{dl}$ for at least 120 minutes. Blood samples were immediately processed and plasma samples frozen at $-28^{\circ} \mathrm{C}$. Plasma pancreatic polypeptide levels were determined with a commercially available radioimmunoassay (Euro-Diagnostica AB, Malmö, Sweden). The 
fasting normal pancreatic polypeptide plasma level for this assay is less than $100 \mathrm{pg} / \mathrm{ml}$.

\section{DATA ANALYSIS}

Sensory thresholds

Mean pressure values during first perception and maximal tolerated pressure were determined for each individual subject. For this purpose, the pressures of the ramps that were associated with the first report of perception or pain were identified. Values for the first and second measurement were compared and the correlation coefficients determined.

\section{Intestinal motility}

After removal of artefacts (simultaneously occurring pressure activities with an up stroke of more than $100 \mathrm{~mm} \mathrm{Hg} / \mathrm{s}$ ), contractions above $5 \mathrm{~mm} \mathrm{Hg}$ were identified and used for further calculations. The last 90 minutes before nutrient infusion, excluding the time segments with duodenal distension, were used to assess interdigestive motility. For assessment of the motor response to nutrients, the mean motility index $(\log [1+(\mathrm{f} \times \mathrm{Amp})])$ of all channels for the 60 minutes before and during nutrient infusion was quantitated. Data analysis was done separately for each channel and averaged for recording sites orad and aborad to the barostat bag. Furthermore, mean values for the intestinal motility index (MI) for the two manometric ports orad and aborad next to the bag were calculated for five minute time periods before and during duodenal distension and an intestinal index of response was calculated (the distension index) by subtracting the MI of the five minute period prior to balloon distension by the MI of the five minute time segment starting with the beginning of the distension.

\section{Small intestinal compliance}

The bag volume was corrected for compressibility according to Boyle's law $\left(\mathrm{P}_{1} \mathrm{~V}_{1}=\mathrm{P}_{2} \mathrm{~V}_{2}\right)$. Duodenal compliance was calculated as the ratio $\mathrm{dV}: \mathrm{dP}$. For this purpose the volume of the

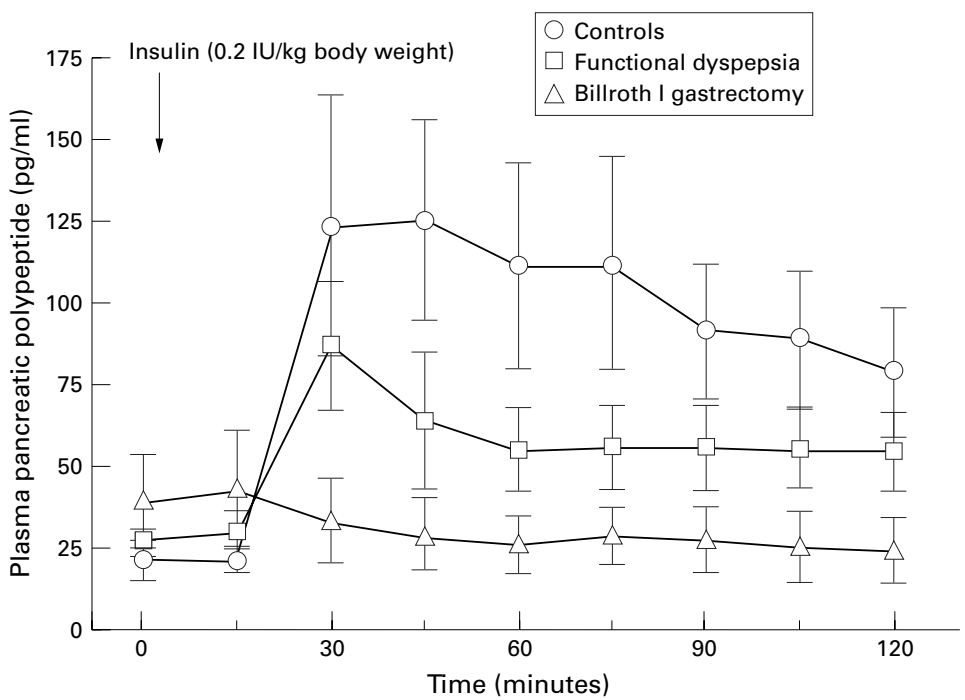

Figure 1 Mean (SEM) plasma PP levels before and after insulin hypoglycaemia in healthy controls $(n=7)$, patients with functional dyspepsia $(F U D, n=7)$ and patients with Billroth I gastrectomy $(B-I, n=6) ; p<0.01$ for the cumulated PP response in controls versus FUD or B-I and $p<0.05$ for functional dyspepsia versus $B-I$. distending bag was assessed at each pressure step, the pressure volume curve determined, and the slope calculated. Compliance is expressed as $\mathrm{ml} / \mathrm{mm} \mathrm{Hg}$.

Pancreatic polypeptide

For plasma PP, the delta between the baseline value before administration of insulin and the highest PP value within 60 minutes after administration of insulin was determined. Based on measurements in healthy controls, an increase in PP of more than $200 \%$ was considered normal.

For all numeric variables, mean values (SEM) or median values if appropriate were calculated.

\section{STATISTICAL ANALYSIS}

Non-parametric tests were used to compare sensory thresholds, PP responses to insulin hypoglycaemia, motor responses to duodenal nutrient infusion, and the motor response to intestinal balloon distension in the different populations. ${ }^{19}$ Fisher's exact test was used to compare frequencies in different groups. In addition, Spearman rank correlations were utilised to assess the association between PP responses to insulin hypoglycaemia and sensory thresholds. All $\mathrm{p}$ values calculated were two tailed; an $\alpha$ level of $5 \%$ was considered significant. Statistical analyses were performed using the Statistical Analysis System. ${ }^{20}$

\section{Results}

BASAL PLASMA LEVELS OF PANCREATIC

POLYPEPTIDE AND RESPONSES TO HYPOGLYCAEMIA

Figure 1 depicts mean PP values before and after insulin administration. Before insulin administration, all PP values were within the normal range and significantly $(p<0.01)$ increased in patients with functional dyspepsia and controls (median delta above baseline $56.7 \mathrm{pg} / \mathrm{ml}$, range $19.8-195.5$, and $162.6 \mathrm{pg} / \mathrm{ml}$, range 34.3-256.6) after insulin administration. The integrated PP response after insulin administration (area under the curve) was significantly $(p<0.01)$ smaller in patients with functional dyspepsia compared with controls while basal PP levels were not significantly different (patients 19.3, range 14.7-25.3 pg/ml; controls 15.7, range $2.8-47.9 \mathrm{pg} / \mathrm{ml}, \mathrm{p}>0.6$ ). Between 15 and 45 minutes after administration of insulin, peaks of PP occurred. Pancreatic polypeptide values increased by more than $200 \%$ in all healthy subjects while five out of seven patients with functional dyspepsia had a PP response to insulin hypoglycaemia that was below the $200 \%$ threshold $(\mathrm{p}<0.05)$.

Pancreatic polypeptide levels in patients after gastrectomy were not significantly altered during insulin hypoglycaemia $(\mathrm{p}>0.6)$ and thus the peak response was significantly smaller compared with healthy controls $(p<0.001)$ and patients with functional dyspepsia $(p<0.001)$. Basal values and values during insulin hypoglycaemia were not different for gastrectomised patients with and without truncal vagotomy (data not shown). 

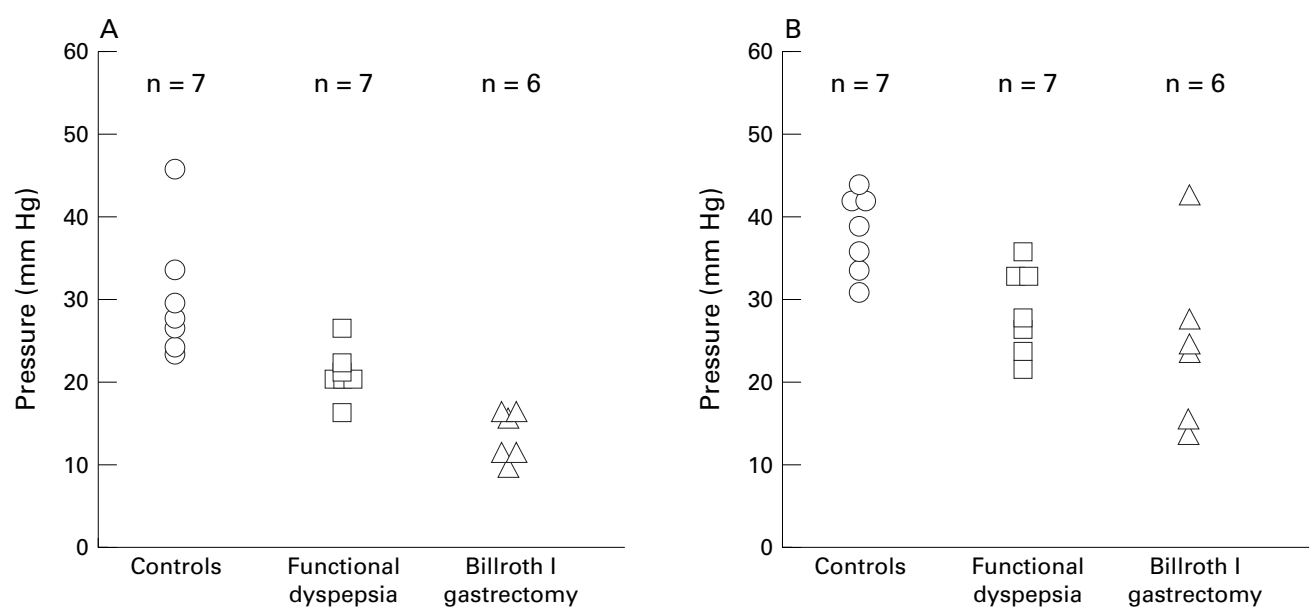

Figure 2 Pressure at first perception (A) and maximal tolerated pain (B). First perception: functional dyspepsia versus controls, $p<0.02$; Billroth I gastrectomy versus functional dyspepsia, $p<0.05$. Maximal tolerated distension: functional dyspepsia versus controls, $p<0.05$; Billroth I gastrectomy versus controls, $p<0.05$.

SENSORY THRESHOLD FOR FIRST PERCEPTION AND MAXIMAL TOLERATED DISTENSION

Perception of intestinal balloon distension occurred at significantly lower pressures in patients with functional dyspepsia compared with healthy controls $(\mathrm{p}<0.02)$. Similarly, pressure thresholds for maximal tolerated distension were significantly $(\mathrm{p}<0.05)$ lower in patients with functional dyspepsia compared with healthy controls. However, thresholds for first perception, but not the maximal tolerated pressure, were even lower $(p<0.05$, fig 2$)$ in patients after Billroth I gastrectomy compared with patients with functional dyspepsia. Pressure thresholds for the two consecutive measurements (first perception or the maximal tolerated distension) were not different (all $\mathrm{p}>0.5$ ) and the consecutive two measurements significantly were correlated (first perception, $r=0.93, \mathrm{p}<0.01$; maximal tolerated distension, $r=0.91, \mathrm{p}<0.01$ ). Age was not associated with sensory thresholds $(\mathrm{p}>0.5)$. Sensory thresholds were not different for gastrectomised patients with or without documented truncal vagotomy and small bowel compliance was not different among these groups (data not shown).

\section{INTESTINAL MOTILITY}

\section{Interdigestive motility}

All controls, four patients with functional dyspepsia, and three patients after Billroth I gastrectomy had at least one propagated phase III before the start of the duodenal nutrient infusion.

\section{Nutrient stimulated motility}

Infusion of nutrients induced a typical postprandial motility pattern with irregular motor activity and absence of duodenal phase III complexes in all patients and healthy controls. Nutrient infusion only slightly increased the intestinal motility index (table 1). However, no significant differences between patients and healthy controls with regard to the duodenal motility index before and during nutrient infusion or the response to intraduodenal nutrients were found. Similarly, the intestinal motor responses in subjects with a normal or impaired response of PP to hypoglycaemia were not significantly different.

Motor response to intestinal balloon distension During distension, in six out of seven healthy controls the motor activity aborad to the distending bag decreased during distension $(p<0.05$ versus before distension). Only in one patient with functional dyspepsia did a decrease in the motor activity occur during distension (fig 3). In contrast, three patients after Billroth I gastrectomy had an increase in the motor activity, two subjects a decrease, and one patient did not show any response to distension. Orad to the distending bag motor activity tended to increase in healthy controls $(p=0.09)$; patients with functional dyspepsia and gastrectomised patients did not show any significant change of duodenal motor activity (all $\mathrm{p}>0.4$ ).

\section{RESPONSE OF PANCREATIC POLYPEPTIDE AND} SENSORY THRESHOLDS

Figure 4 depicts the association between the highest (peak) plasma PP level after administration of insulin and sensory thresholds in healthy controls, patients with functional dyspepsia, and patients after Billroth I gastrectomy. The peak PP level in response to insulin hypoglycaemia was significantly correlated with the pressure at first perception and the maximal tolerated pressure $(r=0.65, \mathrm{p}<0.002$; and $r=0.53, \mathrm{p}<0.02$ ). Analysing patients (functional dyspepsia and Billroth I gastrectomy) and controls separately, the association between the pressure at first perception and the peak PP level yielded significance for patients $(r=0.67, \mathrm{p}<0.02)$ but not controls $(r=-0.28$, $\mathrm{p}>0.5)$.

Table 1 Small intestinal motility index in the different study groups before and during intraduodenal nutrient infusion

\begin{tabular}{llll}
\hline & Controls & $\begin{array}{l}\text { Functional } \\
\text { dyspepsia }\end{array}$ & $\begin{array}{l}\text { Billsoth I } \\
\text { gastrectomy }\end{array}$ \\
\hline Before infusion & $6.66(0.1)$ & $5.95(0.4)$ & $6.34(0.3)$ \\
During infusion & $6.75(0.2)$ & $6.02(0.3)$ & $6.51(0.4)$ \\
\hline
\end{tabular}

Results are expressed as mean (SEM) values across all recording sites. 


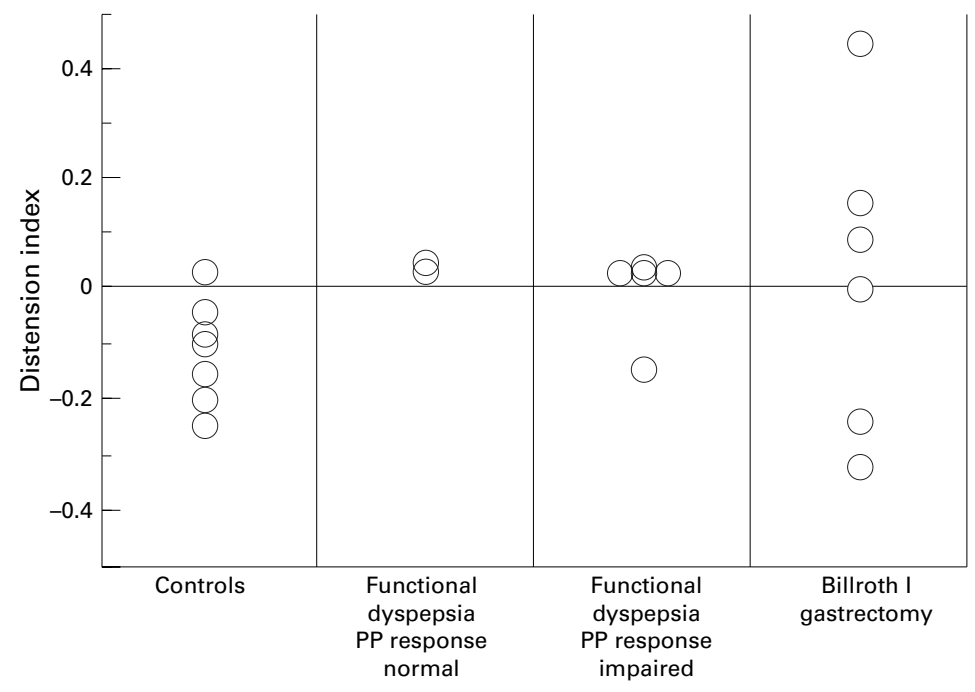

Figure 3 Distension index (MI during distension - MI before intestinal distension).
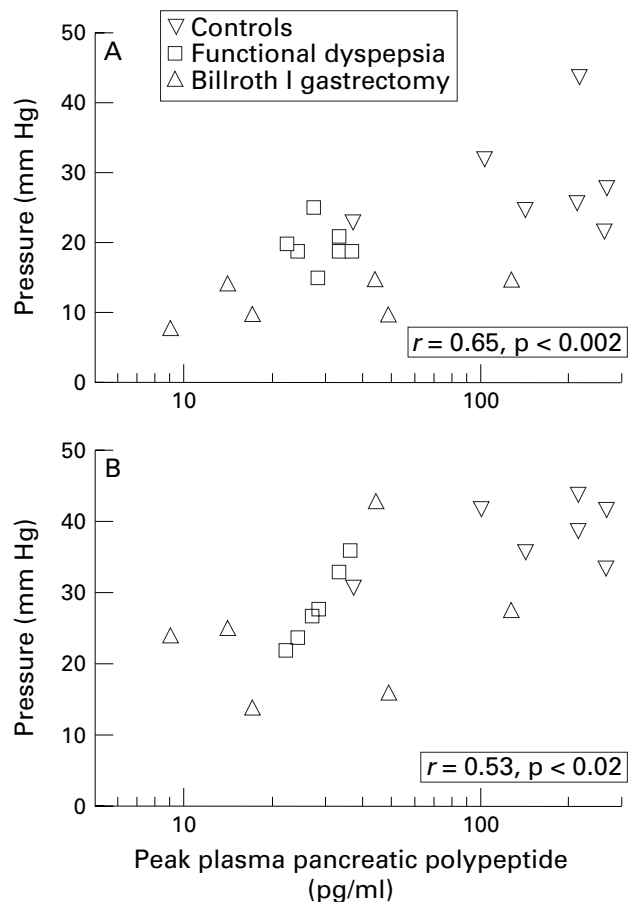

Figure 4 Association between the peak PP level in response to insulin hypoglycaemia and thresholds for first perception $(A)$ and maximal tolerated pressure $(B)$.

\section{Discussion}

Several studies have shown lowered sensory thresholds in at least a proportion of patients with otherwise chronic unexplained dyspepsia (referred to as non-ulcer or functional dyspepsia) and irritable bowel syndrome (IBS). ${ }^{1} 245$ 10-12 Thus, altered mechanosensory function may play a key role in the pathophysiology of functional gastrointestinal disorders. However, very little is known about the mechanisms that modulate sensory function. While past studies have focused on the role of spinal nerves mediating visceral afferents from the gut, ${ }^{21-24}$ it has recently been recognised that vagal afferent fibres may be important in the modulation of nociception. ${ }^{25-27}$ Vagal efferent dysfunction has also been documented in some patients with functional dyspepsia or
IBS. ${ }^{12} 132829$ Camilleri and coworkers found impaired vagal function as assessed by a modified sham feeding test. ${ }^{12}$ Although these investigators also found lowered intestinal thresholds in subjects with dyspepsia and normal small bowel transit, they did not evaluate the association between sensory thresholds and pancreatic polypeptide responses, ${ }^{12}$ and they did not use a barostat with an infinitely compliant balloon in the experiment.

We found that small intestinal visceral sensory thresholds in patients with functional dyspepsia were significantly lower compared with healthy controls, consistent with previous results. ${ }^{10-12}$ Interestingly, in patients with a partial gastrectomy, sensory thresholds were even lower compared with patients with functional dyspepsia. Furthermore, the response of pancreatic polypeptide blood levels to hypoglycaemia was attenuated in a subgroup of patients with dyspepsia and in all patients with a gastrectomy. This suggests that there is impaired vagal function in a subgroup of patients with functional dyspepsia. The pancreatic polypeptide response to hypoglycaemia correlated significantly with the sensory threshold, supporting a role for impaired vagal function in the development of visceral hyperalgesia.

Insulin hypoglycaemia primarily tests vagal efferent function. ${ }^{12}{ }^{30}$ In patients with partial gastrectomy the attenuated pancreatic polypeptide response following partial gastrectomy is almost certainly due to dissection of vagal fibres. There was no difference in sensory thresholds and pancreatic polypeptide responses to hypoglycaemia in gastrectomised subjects with or without a documented truncal vagotomy. Indeed, in animal studies a partial gastrectomy alone has been shown to abolish the pancreatic polypeptide response to electric stimulation of the truncal vagus, indicating vagal transection. ${ }^{31}$

It is estimated that $80 \%$ of the vagal fibres are afferent, conducting signals from the gut to the central nervous system. If the interruption of vagal afferents directly decreases the transmission of visceral mechanosensory information, then this should lead to increased sensory thresholds. However, the opposite was observed: sensory thresholds were lowered in subjects with transected vagal fibres or impaired vagal function as indicated by an attenuated response of the pancreatic polypeptide levels to hypoglycaemia. In line with these findings, previous studies on the effects of proximal selective or truncal vagotomy in patients with functional dyspepsia have failed to show improvement of symptoms after vagotomy; indeed, some patients with functional dyspepsia experienced deterioration in symptoms after vagotomy. ${ }^{32} 33$

Thus, the vagal nerve may not directly mediate visceral mechanosensory information. The observation of an association between disturbed vagal function and lowered sensory thresholds, however, suggests that the vagus does modulate mechanosensory function of the upper gut. The "normal" vagal nerve seems to exert an antinociceptive effect on upper gut mechanosensory function. This antinociceptive effect of the vagal 
nerve is eliminated by interruption of vagal fibres and as a consequence visceral hyperalgesia occurs. In turn this also suggests that in humans visceral sensory function is most likely mediated by spinal pathways ${ }^{6}$ and vagal afferents may directly inhibit the perception of (spinal) mechanosensory information. Indeed, electrical stimulation of vagal afferents decreased the responses of spinothalamic tract neurones to noxious stimulation in rats. ${ }^{34}$

There were no differences among the study populations in relation to the small intestinal motility index before and during nutrient infusion, in contrast to previous studies which showed antroduodenal motor disturbances in patients with functional gastrointestinal disorders. ${ }^{17}$ However, in the present study a liquid meal was infused directly into the duodenum and this may account for the difference.

In summary, the present study has shown altered intestinal visceral sensory function in patients with functional dyspepsia and a Billroth I gastrectomy. Furthermore, a subgroup of patients with functional dyspepsia and all patients with a Billroth I gastrectomy had diminished responses of pancreatic polypeptide to insulin hypoglycaemia, indicating impaired vagal function. As impaired vagal function and lowered visceral sensory thresholds were clearly associated, our data suggest that in humans vagal afferents inhibit visceral sensory information that is transmitted via spinal pathways.

This work was supported by a grant from Deutsche Forschungsgemeinschaft, grant number Ho 1193/3-3. The skilful assistance of Ms J Neufang-Hüber and $\mathrm{Mr} \mathrm{H}$ Fischer is greatly appreciated. Parts of this study were presented at the meeting of the American Gastroenterological Association, May 1996 in San Francisco and published as an abstract (Gastroenterology 1996;110:A680).

1 Lemann M, Dederding JP, Flourie B, et al. Abnormal perception of visceral pain in response to gastric distension in chronic idiopathic dyspepsia. The irritable stomach syndrome. Dig Dis Sci 1991;36:1249-54.

2 Bradette M, Pare P, Douville P, et al. Visceral perception in health and functional dyspepsia. Crossover study of gastric distension with placebo and domperidone. Dig Dis Sci distension with

3 Mearin F, Cucala M, Azpiroz F, et al. The origin of symptoms on the brain-gut axis in functional dyspepsia. symptoms on the brain-gut axis in
Gastroenterology 1991;101:999-1006.

4 Ritchie J. Pain from distension of the pelvic colon by inflating a balloon in the irritable bowel syndrome. Gut 1973;14: 125-32

5 Whitehead WE, Holtkotter B, Enck P, et al. Tolerance of rectosigmoid distension in irritable bowel syndrome. Gastroenterology 1990;90:1187-92.

6 Lembo T, Munakata J, Mertz H, et al. Evidence for the hypersensitivity of lumbar splanchnic afferents in irritable bowel syndrome. Gastroenterology 1994;107:1686-96.

7 Mayer EA, Gebhart GF. Basic and clinical aspects of visceral hyperalgesia. Gastroenterology 1994;107:271-93.

8 De Ponti F, Azpiroz F, Malagelada JR. Reflex gastric relaxation in response to distension of the duodenum. $A m \mathcal{F}$ Physiol 1987;252:G595-601.
9 Coffin B, Azpiroz F, Guarner F, et al. Selective gastric hypersensitivity and reflex hyporeactivity in functional dyshypersensitivity and reflex hyporeactivity in

10 Holtmann G, Goebell H, Talley NJ. Impaired small intestinal peristaltic reflexes and sensory thresholds are independent functional disturbances in patients with chronic unexplained dyspepsia. Am $\mathcal{F}$ Gastroenterol 1996;91:48591.

11 Holtmann G, Goebell H, Talley NJ. Functional dyspepsia and irritable bowel syndrome: is there a common pathophysiological basis? Am f Gastroenterol 1997;92:9549 .

12 Greydanus MP, Vassallo M, Camilleri M, et al. Neurohormonal factors in functional dyspepsia: insights on pathophysiological mechanisms. Gastroenterology 1991;100: 1311-18.

13 Smart HL, Atkinson M. Abnormal vagal function in irritable bowel syndrome. Lancet 1987;ii:475-8.

14 Holtmann G, Goebell H, Talley NJ. Dyspepsia in consulters and non-consulters: prevalence, health-care seeking behavour and risk factors. Eur $\mathcal{F}$ Gastroenterol Hepatol 1994;6: 917-24.

15 Talley NJ, Phillips SF, Melton LJI, et al. A patient questionnaire to identify bowel disease. Ann Intern Med 1989;111: $671-4$.

16 Holtmann G, Goebell H, Holtmann M, et al. Dyspepsia in healthy blood donors: pattern of symptoms and association with Helicobacter pylori. Dig Dis Sci 1994;39:1090-8.

17 Malagelada JR, Stanghellini V. Manometric evaluation of functional upper gut symptoms. Gastroenterology 1985;88: 1223-31.

18 Holtmann G, Talley NJ, Goebell H. Association between $\mathrm{H}$. pylori, duodenal mechanosensory thresholds and small intestinal motility in chronic unexplained dyspepsia. Dig intestinal motility in chroni

19 Kruskal WH, Wallis WA. Use of ranks in one-criterion variance analysis. F Am Stat Assoc 1952;47:583-621.

20 SAS Institute Inc. SAS/STAT user's guide, Release 6.03. Cary, NC: SAS Institute Inc., 1988.

21 Jaenig W, Morrison JFB. Functional properties of spinal visceral afferents supplying abdominal and pelvic organs, with special emphasis on visceral nociception. In: Cervero $\mathrm{F}$, Morrison JFB, eds. Progress in brain research. 67th edn. New York: Elsevier, 1986:87-114.

22 Gebhart GF, Ness TJ. Central mechanisms of visceral pain. Can 7 Physiol Pharmacol 1991;69:627-34.

23 Ness TJ, Metcalf AM, Gebhart GF. Psychophysiological studies in humans using colonic distension as a noxious visceral stimulus [abstract]. Gastroenterology 1990;98:A377.

24 Mayer EA, Gebhart GF. Basic and clinical aspects of visceral hyperalgesia. Gastroenterology 1994;107:271-93.

25 Grundy D, Scratcherd T. Sensory afferents from the gastrointestinal tract. In: Schultz SG, Wood JD, Rauner BB, eds. Handbook of physiology. New York: Oxford University Press, 1989:593-620.

26 Grundy D. Speculations on the structure/function relationship for vagal and splanchnic afferent endings supplying the gastrointestinal tract. F Auton Nerv Syst 1988;22:17580 .

27 Gebhart G, Randich A. Vagal modulation of nociception. Am Pain Soc f 1992;1:26-32.

28 Hausken T, Svebak S, Wilhelmsen I, et al. Low vagal tone and antral dysmotility in patients with functional dyspepsia. Psychosom Med 1993;55:12-22.

29 Haug TT, Svebak S, Hausken T, et al. Low vagal activity as mediating mechanism for the relationship between personality factors and gastric symptoms in functional dyspepsia. Psychosom Med 1994;56:181-6.

30 Feldman M, Taylor IL. Effect of cephalic-vagal stimulation on insulin, gastric inhibitory polypeptide, and pancreatic polypeptide release in humans. $\mathcal{F}$ Clin Endocrinol Metab 1982;55:1114-17.

31 Kameyama J, Suzuki, A, Yasaku Y, et al. Effect of distal gastrectomy on gut hormone release following vagal stimulation in dogs. Eur Surg Res 1993;25:390-5.

32 Aase ST, Liavag I, Roland M. Proximal gastric vagotomy in dyspeptic patients without an ulcer. World $\mathcal{F}$ Surg 1984;8 303-7.

33 Christiansen J, Aagaard P, Koudahl G. Truncal vagotomy and drainage in the treatment of ulcer-like dyspepsia without ulcer. Acta Chir Scand 1973;139:173-5.

34 Ren K, Randich A, Gebhart GF. Effects of electrical stimulation of vagal afferents on spinothalamic tract cells in the rat. Pain 1991;44:311-19. 